$11 \mid 2019$

Migration et exil environnemental : du Déluge à nos jours

\title{
Les jeux de cartes dans le roman libertin du dix- huitième siècle
}

\section{Luisa Messina}

\section{OpenEdition}

Journals

Édition électronique

URL : http://journals.openedition.org/lcc/1562

DOI : $10.4000 /$ lcc. 1562

ISSN : 2430-4247

Éditeur

Université Aix-Marseille (AMU)

Référence électronique

Luisa Messina, « Les jeux de cartes dans le roman libertin du dix-huitième siècle », Les chantiers de la création [En ligne], 11 | 2019, mis en ligne le 20 avril 2019, consulté le 08 avril 2020. URL : http:// journals.openedition.org/lcc/1562; DOI : https://doi.org/10.4000/lcc.1562 


\title{
« Les jeux de cartes dans le roman libertin du dix-huitième siècle »
}

\author{
Luisa Messina \\ Université de Palerme \\ luisamess84@libero.it
}

Résumé : Les romans libertins se focalisent traditionnellement sur la scène de séduction. Ils témoignent des jeux principaux répandus au dix-huitième siècle et relatent des histoires qui ont lieu dans un milieu social bien précis. Après la mort de Louis XIV, un nouveau mythe, né dans les années de la Régence, inaugure le siècle libertin en jetant les bases d'une société animée des spectacles, du luxe, de l'art voluptueux et de la richesse. La noblesse oisive aime s'amuser en participant aux spectacles théâtraux et en jouant pour éviter l'ennui. Quelques détails, parsemés dans les descriptions littéraires, nous permettent de comprendre que les jeux de cartes figurent parmi les jeux préférés de l'aristocratie du dix-huitième siècle.

Mots-clés : roman, libertin, dix-huitième siècle, jeu, cartes.

\begin{abstract}
Libertine novels are traditionally focused on seduction scene. They show some games They testify main games in the eighteenth century because they set in a very specific social ambiance. After Louis XIV's death, a new myth, born during the Regence, initiates libertine century laying the foundations of a society which is animated by shows, luxury, voluptuous art and richness. Idle nobility loves entertaining, going to the theatre and playing to avoid being annoying. Some details, scattered in descriptions, allow to understand what are main games such as cards.
\end{abstract}

Keywords : novel, libertine, Eighteenth century, game, card.

Les romans libertins du dix-huitième siècle focalisent l'attention sur le récit de séduction. Mais on trouve aussi quelques descriptions parsemées qui nous permettent de constater la diffusion des jeux. Après la mort du Roi Soleil, on constate la naissance d'un mythe qui, né dans les années de la Régence, inaugure le siècle libertin en jetant les bases d'une société fondée sur l'esthétique du plaisir, dominant le siècle des Lumières, qui passe à travers l'érotisme, la nourriture, le luxe et les divertissements les plus variés. À l'aube de la Révolution, la noblesse oisive aime s'amuser en participant aux spectacles et en jouant pour échapper de l'ennui quotidien. Quelques œuvres 
libertines sont donc fondamentales pour mener des analysesussi bien littéraires qu'historiques sans oublier l'impact sociologique. Les écrits qui seront pris en compte dans cet article témoignent non seulement de l'importance sociale des jeux, mais aussi des symptômes d'une classe sociale débauchée destinée au coucher à cause de son attitude de plus en plus superficielle, mondaine et corrompue.

En général, le jeu est conçu comme l'un des principaux divertissements d'une société dont le seul souci est l'amusement. On relève notamment des références concernant les jeux de cartes à la lecture et l'analyse des romans libertins les plus significatifs du dix-huitième siècle. En effet, les jeux de hasard (dés et cartes) comme tous les jeux de table ne nécessitent qu'un matériel facile à transporter. Les jeux de cartes font partie de la vie mondaine de la classe aisée et finissent par se répandre à la cour de Versailles ainsi qu'à la capitale ${ }^{1}$ En effet, la seconde moitié du dix-septième et le dix-huitième siècle sont marqués par le succès des salons ou des académies de jeux où triomphent les jeux de cartes. À Paris bien au moins huit académies de jeux existent, qui sont constamment contrôlées par des espions au service du lieutenant de police, et un certain nombre de tripots tenus par des ambassadeurs, des princes étrangers vivant à Paris et ainsi que par quelques protagonistes de la haute noblesse de la cour : le tripot du prince de Transylvanie est l'un des plus célèbres du dixhuitième siècle en raison de ses parties de pharaon ${ }^{2}$. Toutes ces personnalités ont le permis de tenir table pour des jeux de hasard (Freundlich 27-29). On sait que vers 1750 quatre sociétés organisées par Madame de Monasterol, la marquise de Berville, Madame de Vogüé et Madame d'Estillac invitent de nombreux nobles venant de grandes familles du royaume : Sade, Valentinois, Tessé, Grammont, Maillebois, Villers, d'Antin, Brissac ne sont que les plus connus. Le comte de Genlis gaspille en une nuit les rentes de plusieurs familles (Roche 12).

Les autorités de l'Ancien Régime ont leurs raisons pour réprimer les jeux dans les maisons royales ainsi que chez les limonadiers. Le jeu représente une manifestation du désordre opposé à l'idéal de l'ordre : mépris, provocation, gaspillage, frivolité, irréligion (Dunkley, 1976, 14). Même si la police punit les joueurs de la rue et les tenants de loteries clandestines, elle ne réussit pas à contrôler d'une manière efficace ce qui est un phénomène de masse, touchant le peuple aussi bien

\footnotetext{
${ }^{1}$ La première moitié de l'ère moderne est marquée par l'invasion des cartes à jouer. Venant du Moyen et de l'Extrême Orient, elles se répandent en Italie d'abord, en Espagne ensuite, en France enfin au milieu du quatorzième siècle, cf Elisabeth Belmas, Jouer autrefois : essai sur le jeu dans la France moderne (XVI $I^{-} X V I I I^{e}$ siècle) (Seyssel : Champ Vallon, 2006), p.140. À partir de ce moment, leur succès est immédiat. Pourtant, la diversification des jeux de cartes n'aura lieu qu'à partir du XV $\mathrm{V}^{\mathrm{e}}$ siècle, cf Jean-Michel, Mehl, Les jeux au royaume de France du XIII au début du XVI siècle (Paris : Fayard, 1990), p.159.

${ }^{2}$ Le roi autorise le prince de Carignan et le duc de Gesvres à tenir des assemblées de jeux de hasard. L'hôtel de Soissons représente l'un des hauts lieux de l'agiotage sous la Régence tandis que le duc de Gesvres gagne 130.000 livres par an. Tolérées par le roi, les deux académies sont surveillées en permanence par les espions de la police. Pourtant, à la nouvelle de la mort du prince de Carignan (1741), le cardinal de Fleury en profite pour fermer la maison de jeux du prince de Carignan et pour faire cesser le jeu de M. le duc de Gesvres, cf Francis Freundlich, Le monde du jeu à Paris (Paris : Albin Michel, 1995), p.29-30.
} 
que les élites. Cet échec témoigne d'une difficulté à résoudre la contradiction fondamentale existant entre la condamnation publique du jeu et la recherche des ressources nécessaires à l'État. En fait, deux ordonnances royales en 1717 et 1781 interdisent les jeux de hasard mais elles finissent par effrayer les sujets tandis que les grands seigneurs continuent à jouer impunément (Roche 10-11). Dès 1776 les ambitions fiscales de l'État poussent le roi Louis XV à ordonner la création de la Loterie royale de France. C'est Casanova qui propose la création d'une loterie conforme à celle de Gênes, Rome et Milan. Après l'acceptation de son projet, un arrêt du Conseil du roi autorise le principe d'une loterie à raison d'un tirage mensuel. Cette institution fonctionne si bien qu'on crée la Loterie royale de France où l'État se réserve le monopole exclusif de ce jeu. La Loterie royale ensuite rejoint de principales villes françaises en intéressant des couches sociales urbaines (Freundlich 39).

L'analyse de la littérature française du dix-huitième siècle montre que des romans tels que Manon Lescault témoignent du goût immodéré pour les jeux, considérés comme des divertissements mondains frivoles pour la société française de l'Ancien Régime. Les romanciers commencent à prendre leurs distances avec cette passion effrénée comme Montesquieu dans la lettre LVI des Lettres persanes $(1721)^{3}$ et Fougeret de Monbron au début de La capitale des Gaules ou La nouvelle Babylone (1760) ${ }^{4}$.

Les écrits libertins se déroulent dans un contexte social bien reconnaissable où les jeux s'insèrent parfaitement parmi les plaisirs légaux ou illégaux, à la fois tolérés ou réprimés. Les romans libertins nous dévoilent les joueurs en prévalence des femmes aisées ainsi que les principaux jeux de cartes de l'époque comme le pharaon et le médiateur. Il semble que les femmes aient une place prépondérante. Dans le roman Le colporteur (1761) Chevrier propose un véritable tableau des mœurs du siècle en dévoilant la passion effrénée du jeu chez les aristocrates de l'époque. Le

\footnotetext{
${ }^{3}$ Usbek écrit une lettre à son ami et déplore la condition de jouer, qui élève un homme au rang d'homme de qualité et une femme qui finit par ruiner son mari : «Le jeu est très en usage en Europe : c'est un état que d'être joueur ; ce seul titre tient lieu de naissance, de bien, de probité : il met tout homme qui le porte au rang des honnêtes gens, sans examen, quoiqu'il n'y ait personne qui ne sache qu'en jugeant ainsi, il s'est trompé très souvent ; mais on est convenu d'être incorrigible. Les femmes y sont surtout très adonnées. Il est vrai qu'elles ne s'y livrent guère dans leur jeunesse que pour favoriser une passion plus chère ; mais, à mesure qu'elles vieillissent, leur passion pour le jeu semble rajeunir, et cette passion remplit tout le vide des autres. Elles veulent ruiner leurs maris et, pour y parvenir, elles ont des moyens pour tous les âges, depuis la plus tendre jeunesse jusque à la vieillesse la plus décrépite : les habits et les équipages commencent le dérangement ; la coquetterie l'augmente ; le jeu l'achève. », Charles-Louis de Montesquieu Secondat, Lettres persanes, dans CEuvres complètes de Montesquieu, t. II (Paris : Hachette, 1859), p.189-190.

${ }^{4}$ D'après Fougeret de Monbron les jeux ont abattu les barrières sociales et ont rendu honorable un divertissement une fois déplorable : «Les jeux font à Paris du grand secours pour quiconque n'a rien et n'est propre à rien. Ils tiennent lieu de patrimoine, d'office et de charges. Ils rapprochent toutes les conditions et mettent une sorte d'égalité parmi les grands et les petits, les gens d'esprit et les autres sors. On rougissait autrefois du métier de joueur ; aujourd'hui c'est une profession recommandable et qui donne entrée partout. Il y a dans Paris, à la honte du bon ordre, deux cents maisons de jeux, ou plutôt deux cents coupe-gorges qui sont le rendez-vous des filous et des dupes », Louis-Charles Fougeret de Monbron, La capitale de Gaule ou la nouvelle Babylone (Paris, 1760), p.14.
} 
romancier nous explique comment fonctionnent les tripotières, mot indiquant les femmes qui tiennent un tripot ${ }^{5}$ :

Une de ces dames, que l'on nomme à Paris tripotière, du nom avilissant des assemblées qu'elles tiennent, réunit trois ou quatre personnes en fonds, qui, formant entre elles une somme de cinq cents louis, font valoir l'argent sans qu'elles paraissent. La banque une fois établie, on cherche un tailleur, c'est-à-dire un homme qui, tenant des cartes, a le secret de les connaître au tact, et de filer celles qui lui sont nuisibles (Chevrier, 1993, 90).

Il est évident que l'écrivain lorrain, dont la veine satirique est notoire à l'époque, tourne en dérision la société mondaine parisienne dominée par des dames frivoles qui se consacrent au plaisir et au divertissement. Il n'hésite pas à nommer les femmes dominées par le jeu comme la duchesse de Phalaris, une des nombreuses maîtresses du régent Philippe d'Orléans, qui désormais mène une vie frivole en jouant au pharaon. Celui-ci est un jeu de cartes qui est particulièrement en vogue à la cour de Versailles, sous les règnes de Louis XV et de Louis XVI. Il se joue entre un banquier et un nombre illimité de joueurs: les règles de ce jeu sont telles qu'elles avantagent de beaucoup le banquier. Celui-ci gagne la mise du ponte, lorsque la carte du ponte arrive dans la main droite et de rang impair, et il perd, lorsque la carte du ponte tombe dans la main gauche, et de rang pair (Collectif, 341). Une partie de pharaon permet à la duchesse d'évoquer le temps passé avec son ancien amant :

Les femmes qui embrassent ce train de vie n'ont d'autre occupation que se donner à jouer ; telles sont aujourd'hui la vicomtesse de $\mathrm{P}^{* * *}$ la marquise de $\mathrm{M}^{* * *}$, et l'éternelle duchesse de Pha*** qui n'a plus pour elle qu'une table de pharaon et le souvenir des plaisirs qu'elle goûta avec le duc d'Orléans, régent du royaume. Ces femmes vivent exactement du profit du jeu (Chevrier, 1993, 889-890).

Cette citation permet aussi de rappeler ce que Diderot a déjà observé dans Les bijoux indiscrets (1748). Le sultan philosophe, héros du roman, se demande pourquoi les femmes agissent de manière si fébrile lors d'une partie de pharaon, au point de parler de « fureur » :

La passion du jeu est une des moins dissimulées ; elle se manifeste, soit dans le gain, soit dans la perte, par des symptômes frappants. "Mais d'où leur vient cette fureur? se disait-il en lui-même; comment peuvent-elles se résoudre à passer les nuits autour d'une table de pharaon, à trembler dans l'attente d'un as ou d'un sept ? Cette frénésie altère leur santé et leur beauté, quand elles en ont, sans compter les désordres où je suis sûr qu'elle les précipite (Diderot 72).

\footnotetext{
${ }^{5}$ Le mot « tripot » désigne une sorte de maison de jeux, créée par quelques dames de l'époque.
} 
Diderot va plus loin que Chevrier dans la mesure où il observe que la passion immodérée pour le jeu risque d'altérer la santé physique et morale des femmes ${ }^{6}$.

Si les femmes excellent dans le jeu, les hommes aussi sont très assidus. Dans Les égarements du cour de l'esprit (1736) Crébillon montre l'entrée dans le monde d'un jeune homme qui, avant de rencontrer son mentor Versac, méconnaissait les divertissements sociaux tels que les jeux de cartes. Meilcour est obligé de jouer à une partie d'hombre, un jeu de cartes espagnol très en vogue en France au dix-huitième siècle (Belmas, 403-404). Malgré son inexpérience juvénile, le jeu lui donne l'occasion d'admirer la demoiselle qu'il convoite :

Madame de Lursay [...] me mit avec Madame de Théville contre Madame de Senanges et contre lui, et fit une reprise d'hombre! avec Hortense et Monsieur de Pranzi. Dans le chagrin que j'en eus, je pensais rompre la partie que je venais d'accepter. Pour m'en dédommager du moins, je me plaçais de façon que j'avais Mademoiselle de Théville en face : pénétré du plaisir de la regarder, je ne sus pas un instant ce que je faisais.

[...] Le jeu ne nous intéressait pas assez pour nous tenir dans le silence (Crébillon 92).

Ce passage est assez significatif de ce que les jeux représentent dans la bonne société du dixhuitième siècle. Il est évident que les jeux font partie d'un cérémoniel social qui suscite différentes attitudes parmi les participants. Ainsi, le jeune Meilcour est-il d'abord déconcerté face au jeu. Mais cette inquiétude ne dure que quelques instants parce que le jeune homme y trouve une opportunité pour observer la belle Hortense ${ }^{7}$. Étant plus familiers des usages mondains, Versac et Madame de Senanges profitent du jeu pour discuter d'autres affaires comme de nouvelles bagatelles. En revanche, leur attitude impatiente Madame de Théville qui «aimait le jeu comme une femme qui n'aime pas autre chose » (Crébillon 92).

Dans Les confessions du comte de *** (1742), Duclos décrit le parcours d'un libertin débauché qui passe des bouleversements de la vie mondaine parisienne à la tranquillité des plaisirs agrestes. Le comte libertin en question est en effet invité chez Madame de Tonins dont le salon est

\footnotetext{
${ }^{6}$ De la même manière que Les bijoux indiscrets, les jeux apparaissent aussi dans les contes de fées apparemment orientaux qui, apparus à la moitié du dix-huitième siècle, finissent pour dévoiler les excès caractérisant la France de l'époque. Par exemple, Ma-gakou, l'un des trois contes orientaux de François-Antoine Chevrier, atteste de la présence de certains jeux vieux comme Partie de tri qui se réfère à un jeu d'hombre pour trois joueurs où on élimine le carreau sauf le roi. Pour échapper à l'ennui, le jeune Ma-gakou manifeste sa prédilection pour le jeu parce qu' « [...] il préféra une partie de tri, jeu renouvelé des Sylphes », François-Antoine Chevrier, Ma-gakou: histoire japonaise, (Paris, 1752), p. 141.

Lors de sa carrière libertine, Meilcour comprend la valeur du jeu comme une approche visuelle ainsi qu'une opportunité pour cueillir les signes d'intérêt à travers l'observation de la face et des gestes d'autre personne comme dans le cas de la partie prise ici en considération cf John Dunkley, « Gambling : a social and moral problem in France » (Studies on Voltaire, $\mathrm{N}^{\circ} 235,1985$ ), p.200. Comme l'observe T. Kavanagh, la séduction et le jeu servent à ajouter à la convivialité une dimension de suspense qui redéfinit la manière où les hommes et les femmes sont liés. Ce procédé est observable chez Crébillon et, en particulier dans son roman Le hasard du coin $d u$ feu (1763), où Célie rencontre son premier amour lors d'une partie de cartes, cf Thomas Kavanagh, « The libertine's bluff. Card and cultures in eighteenth century France » (Eighteenth-Century Studies, Vol. 33, № 4, 2000), p. 507-508.
} 
plein de beaux esprits et de gens du monde. Pourtant, le jeu devient une activité nécessaire au moment où les sujets de conversation sont épuisés :

Au bout d'une heure je m'aperçus que la conversation languissait; je proposai une partie de jeu, moins par goût que par habitude de voir jouer. Madame de Tonins me dit que le jeu était absolument banni de chez elle, qu'il ne convenait qu'à ceux qui ne savaient ni penser, ni parler. "C'est, ajouta-t-elle, un amusement que l'oisiveté et l'ignorance ont rendu nécessaire ». Ce discours était fort sensé ; mais malheureusement Madame de Tonins et sa société malgré tout leur esprit souvent dans le cas d'avoir besoin du jeu, et ils éprouvaient que la nécessité d'avoir toujours dans l'esprit est aussi importune que celle de jouer toujours. Le jeu devint la matière d'une dissertation qui dura jusqu'au soupe (Duclos 221-2).

Malgré les réticences de la sage Madame Tonins, dont la justesse de raisonnement à propos du jeu est reconnue par le protagoniste débauché, il semble que le jeu soit le seul expédient qui permette aux hommes d'esprit de s'amuser et d'oublier quelques instants des argumentations trop sérieuses.

Dans Thémidore (1744) Godard d'Aucour mentionne le jeu du médiateur qui est un jeu de cartes très à la mode, même toléré par la police. Ce divertissement suscite l'attention du marquis d'Argenson qui constate que la nouvelle pretintaille de quadrille [les nouvelles règles du jeu de cartes] intitulée le médiateur a été instituée afin de tourner en dérision la personnalité du cardinal de Fleury qui prétend être le médiateur universel de l'Europe [...] (Voyer d'Argenson 207). Or, le jeune Thémidore joue au médiateur lors d'une soirée consacrée aux plaisirs qui comprend aussi un riche souper :

On se leva de table. Après quelques tours du jardin on fit un médiateur. Le président gagna beaucoup. Il jouait d'un bonheur sans égal. Rozette en était outrée : ce n'est pas aux cartes où elle est belle joueuse, elle nous répéta souvent qu'elle était un péché mortel, parce qu'elle ne voyait pas un as noir. Cependant elle trichait suivant le talent qu'elle avait reçu. Argentine, que je conseillais, l'imitait au mieux. Le président s'en apercevait et en riait sous cape ; il sait comme vous et moi que toute femme triche, et que même lorsqu'elles veulent être fidèles, l'habitude supplée à leur intention (Godard d'Aucour 515-6).

Rozette, la femme que Thémidore essaie de séduire, est d'habitude une bonne joueuse parce qu'elle triche de la même manière que son amie Argentine. Thémidore et son ami président laissent libre cours à ces tricheries pour passer une bonne soirée en compagnie de belles courtisanes.

Dans Ma-gakou (1752) Chevrier n'écarte pas la possibilité de s'attacher aux hommes qui, apparemment agréables, gagnent de l'argent au jeu bien qu'ils ne possèdent ni qualités ni moyens : 
Les maisons ouvertes aux jeux, celles même des gens en place, sont infestées de ces hommes brillants et gracieux, dont le commerce n'est dangereux que parce qu'il est doux ; de ces êtres errants qui, sans patrie, sans moyen et sans asile, sont reçus sous le titre de joueur. Là toutes les qualités sont confondues, et la bonne foi n'y périt que trop souvent sous les coups d'une fortune industrieuse. On comptait autrefois les fripons au jeu, bannis des villes, ils étaient l'opprobre de la société, aujourd'hui tous les cercles en sont remplis, et l'impunité dans laquelle ils vivent me fait trembler sur l'avenir (Chevrier, 1752, 121-2).

Les romans les plus célèbres de Chevrier, les Mémoires d'une honnête femme (1753) et Le colporteur (1761), finissent par confirmer la vérité des jeux de cartes entre les aristocrates. On comprend que les jeux font désormais partie des divertissements sociaux de la même manière que les repas et les spectacles.

Julie, la protagoniste du roman Mémoire d'une honnête femme (1753), se déplace avec son mari à Paris où elle fait la connaissance des gens du grand monde qui ne poursuivent que les divertissements les plus frivoles comme des jeux de cartes : «On proposa une partie de manille, c'était alors le jeu à la mode, que les petites-maitresses viennent de renouveler sous le nom de comète $^{8}$; je la fis, et l'arrangement de la Baronne de Verman me mit en fac du Comte qui, de la dignité qui ne lui réussit point, passa au plaisant qui ne prit pas plus» (Chevrier, $2005: 39$ ).

Si l'on analyse le passage cité, Chevrier mentionne la manille et la comète, deux jeux qu'il indique en italique. La manille à laquelle Chevrier fait référence peut être soit à ce que l'on appelle aussi la « sizette » soit à ce que les académies universelles de jeux considèrent comme un synonyme d'une ancienne forme de comète ${ }^{9}$. Après le souper, la compagnie alors s'amuse en jouant du piquet : « Le Chevalier faisait pendant cette conversation un piquet avec le Baron de Verman » (Chevrier, 2005 : $40)^{10}$.

\footnotetext{
${ }^{8}$ Le jeu de la comète est aussi présent dans Ma-gakou quand le jeune protagoniste est accueilli dans la maison d'une coquette où on lui proposer de jouer : « Arrivés tous les trois à la petite maison de l'amant de Delaniga, Ma-gakou se jeta aux genoux de Bazika (sa coquette s'appelait ainsi) et voulut que le plaisir celât son bonheur, mais l'instant n'était pas encore arrivé, et les préliminaires n'étant point remplis, on arrêta l'empressement du voyageur, et on proposa une partie de comète en attendant qu'on servît ». François-Antoine Chevrier, Ma-gakou : histoire japonaise, (Paris, 1752), p.44.

${ }^{9}$ La sizette est un jeu de cartes où six joueurs sont répartis en deux équipes de trois en désignant un gouverneur par chaque équipe. On distribue trente-six cartes, la dernière indique l'atout. La particularité de la sizette est que les joueurs s'informent sur les cartes qu'ils possèdent. Le jeu est gagné par ceux qui font plus tôt trois levées. Cf Nouveau manuel complet des jeux de calcul et de hasard ou Nouvelle Académie des jeux, (Paris : Roret, 1853), p.256-257.

En ce qui concerne le jeu de la manille, considérée comme une forme ancienne de comète, est un des jeux aimés par Louis XIV et Louis XV. On joue à la manille depuis deux personnes jusqu'à cinq. Il est bon de jouer à la suite les cartes de même couleur en commençant par la moindre carte de la suite pour monter jusqu'au roi. Cf Nouveau manuel complet des jeux de calcul et de hasard ou Nouvelle Académie des jeux, (Paris : Roret, 1853), p.270-271.

${ }^{10} \mathrm{On}$ atteste ce jeu de cartes depuis quelques siècles. Il est beaucoup aimé étant donné qu'il permet de jouer à deux ou à quatre personnes auxquelles on distribue douze cartes chacun. On envisage des combinaisons en suivant l'ordre : point, séquence, carte de même rang. On dit qu'il est l'un des jeux les plus appréciés de l'époque. Cf Nouveau manuel complet des jeux de calcul et de hasard ou Nouvelle Académie des jeux, (Paris : Roret, 1853), p.273-274.

«Ceux qui savent le jeu de piquet conviennent que c'est un des plus beaux jeux qui se jouent aux cartes, et dont l'ont ne s'est jamais laissé, comme de la plupart des autres qui ne sont en règne qu'un certain temps, après quoi ils tombent $»$, cf. Edmond Hoyle, François Danican Philidor, Académie universelle des jeux, t. I (Lyon : Cormon et Blanc, 1805 ), p. 1.
} 
Le roman Le colporteur (1761) offre un tableau des mœurs du siècle concernant aussi la passion du jeu entre les aristocrates de l'époque. La duchesse de Phalaris, l'une des nombreuses maîtresses du régent Philippe d'Orléans, mène sa vie en jouant à pharaon et en se souvenant de ses passions passées :

Les femmes qui embrassent ce train de vie n'ont d'autre occupation que se donner à jouer ; telles sont aujourd'hui la vicomtesse de $\mathrm{P}^{* * *}$ la marquise de $\mathrm{M}^{* * *}$, et l'éternelle duchesse de Pha*** qui n'a plus pour elle qu'une table de pharaon et le souvenir des plaisirs qu'elle goûta avec le duc d'Orléans, régent du royaume. Ces femmes vivent exactement du profit du jeu. (Chevrier, 1993, $889-90)^{11}$

Il est évident qu'à travers le personnage du colporteur Chevrier s'en prend à ce type de société mondaine dont les seuls soucis ne sont que le divertissement et l'argent.

La passion du jeu demeure jusqu'à la fin du siècle. Dans Les liaisons dangereuses (1782), Laclos présente plutôt la ruse de la marquise de Merteuil et explique comment fonctionne ce jeu dans une note placée en bas de page à l'attention du lecteur ${ }^{12}$. La marquise organise une partie de macédoine pendant une belle soirée mondaine pour se venger de Prévan qui est connu comme un grand joueur :

À minuit, les parties étant finies, je proposai une courte macédoine. J'avais le double projet de favoriser l'évasion de Prévan, et en même temps de la faire remarquer; ce qui ne pouvait pas manquer d'arriver, vu sa réputation de joueur. [...]

Le jeu dura plus que je n'avais pensé. [...] J'ai eu la force de résister. Je rebroussai chemin, et revins, non sans humeur, reprendre place à ce jeu éternel. Il finit pourtant, et chacun s'en alla. (Laclos 239).

La marquise de Merteuil explique à Valmont comment elle défie Prévan avant de le tromper en organisant une partie de macédoine comme étape vers sa ruine: sa chute doit avoir lieu publiquement pour satisfaire son orgueil de femme outragée.

Pour ce qui est du jeu Pour ce qui est du jeu, plus ou moins toléré par la monarchie et de manière différente selon ses exigences, il apparaît comme l'une des distractions favorites des aristocrates caractrérisés par la lâcheté/décrits comme lâches., il est alors évident qu'il, plus ou moins toléré par

\footnotetext{
${ }^{11}$ Le pharaon est un jeu de cartes qui se joue entre un banquier et un nombre illimité de joueurs. Il est particulièrement en vogue à la cour de Versailles à la cour de Louis XV et de Louis XVI. Les règles de ce jeu sont telles qu'elles avantagent de beaucoup le banquier qui gagne la mise du ponte, lorsque la carte du ponte arrive à la main droite dans un rang impair, et il perd, lorsque la carte du ponte tombe à la main gauche, et dans un rang pair, cf. Nouveau manuel complet des jeux de calcul et de hasard ou Nouvelle Académie des jeux (Paris : Roret, 1853), p.341.

12 «Quelques personnes ignorent peut-être qu'une macédoine est un assemblage de plusieurs jeux de hasard, parmi lesquels chaque coupeur a droit de choisir lorsque c'est à lui de tenir la main. C'est une des inventions du siècle », Pierre-Ambroise-François Choderlos de Laclos, Les Liaisons dangereuses (Paris : Gallimard, 2012), p.239.
} 
la monarchie et diversifié selon les exigences, est l'une des distractions les plus agréables des aristocrates lâches. En particulier, Chevrier prend ses distances avec la uperficialité de ces aristocrates appartenant aux deux sexes. Tout en faisant partie de la noblesse, Chevrier met l'accent sur les défauts d'une classe sociale destinée à périr avec l'Ancien Régime. À la suite de ces considérations socio-littéraires, il s'avère que les jeux constituent l'un des divertissements les plus en vogue dans la bonne société à la veille de la Révolution. Malgré les ordonnances royales interdisant les jeux de hasard, les nobles français et étrangers se sentent autorisés à tenir des maisons de jeux. D'une part, le jeu fait partie des amusements qui garantissent les échanges sociaux au sein des classes aisées (le personnage du comte de ${ }^{* * *}$ ) de la même manière que les spectacles. D'autre part, les femmes sont les principales initiatrices et organisatrices des jeux au point d'en arriver à des comportements quasi pathologiques comme le dénoncent Diderot qui parle de «fureur» ou Montesquieu ${ }^{13}$. Par touches subtiles, parfois de manière anecdotique, les romans libertins décrivent la stratégie qui accompagne les jeux de cartes. Ainsi, les dames se servent du jeu dans leurs manœuvres de séduction (Madame de Lursay), mais peuvent également le transformer en instrument de vengeance (la marquise de Merteuil). Les écrits libertins, pourtant enclins à une certaine libération des mœurs, formulent aussi une critique face à cette passion effrénée pour les jeux, lesquels sont capables de ruiner une réputation, une fortune, voire des familles entières.

\section{Ouvrages cités}

Argenson Voyer (de), René-Louis. Mémoires et journal inédit du marquis d'Argenson, t. II. Paris : Jannet, 1857.

Belmas, Elisabeth. Jouer autrefois : essai sur le jeu dans la France moderne (XVI'-XVIII siècle). Seyssel : Champ Vallon, 2006.

Chevrier, François-Antoine. Ma-gakou: histoire japonaise, Paris, 1752.

(—.). Le colporteur. Romans libertins du XVIII siècle. Paris : Laffont, 1993.

\footnotetext{
${ }^{13}$ Un homme d'esprit est celui qui est capable d'amuser une dame en jouant comme le témoigne Chevrier avec ironie : « Propose-t-on à une dame du bel air de lui présenter un homme de mérite ? Joue-t-il ? Voilà la réponse ordinaire. Si l'homme de mérite est assez sage pour ne pas jouer, il n'est bon à rien, et on ne le reçoit que comme un de ces importuns, avec lesquels la bienséance veut qu'on s'ennuie une heure par mois ", François-Antoine Chevrier, $M a-$ gakou : histoire japonaise (Paris, 1752), p.141. R. Mauzi considère le jeu comme une réponse parmi d'autres au désir de bonheur qui anime l'homme : Robert Mauzi, «Écrivains et moralistes du XVIII siècle devant les jeux de hasard » (Revue des sciences humaines, $\left.\mathrm{N}^{\circ} 90,1958\right)$, p.225.
} 
(—.). Mémoires d'une honnête femme écrits par elle-même. Saint-Étienne : Publications de l’Université de Saint-Étienne, 2005.

Crébillon, Claude. Les égarements du cour et de l'esprit. Romans libertins du dix-huitième siècle. Paris : Laffont, 1993.

Diderot, Denis. Les bijoux indiscrets. Paris : Gallimard, 1981.

Duclos, Charles-Pinot. Les confessions du comte de ***. Romans libertins du dix-huitième siècle. Paris : Laffont, 1993.

Dunkley, John. «Le jeu de hasard et la loi ». Le jeu au dix-huitième siècle. Aix-en-Provence : Edisud, 1976.

(—.). « Gambling : a social and moral problem in France ». Studies on Voltaire, N 235, 1985 : 188-217.

Fougeret de Monbron, Louis-Charles. La capitale de Gaule ou la nouvelle Babylone. Paris, 1760.

Freundlich, Francis. Le monde du jeu à Paris. Paris : Albin Michel, 1995.

Godard d'Aucour, Claude. Thémidore. Romanciers libertins au XVIII siècle, t, I. Paris : Bibliothèque de la Pléiade, Gallimard, 2000.

Hoyle, Edmond, Philidor François. Académie universelle des jeux, t. I. Lyon : Cormon et Blanc, 1805.

Kavanagh, Thomas. "The libertine's bluff. Card and cultures in eighteenth century France ». Eighteenth-Century Studies, Vol. 33, N 4, 2000 : 505-521.

Laclos Choderlos (de), Pierre-Ambroise-François. Les liaisons dangereuses. Paris : Gallimard, 2012.

Mauzi, Robert. «Écrivains et moralistes du XVIII ${ }^{\mathrm{e}}$ siècle devant les jeux de hasard ». Revue des sciences humaines, $\mathrm{N}^{\circ}$ 90, $1958: 219-256$.

Mehl, Jean-Michel. Les jeux au royaume de France du XIII au début du XVI siècle. Paris : Fayard, 1990.

Montesquieu Secondat (de), Charles-Louis. Lettres persanes, dans Euvres complètes de Montesquieu, t. II. Paris : Hachette, 1859.

Nouveau manuel complet des jeux de calcul et de hasard ou Nouvelle Académie des jeux. Paris : Roret, 1853.

Roche, Daniel. « Préface ». Le monde du jeu à Paris. Paris : Albin Michel, 1995. 